\title{
HACIA LA INVESTIGACIÓN EN DOCENCIA: ORIENTACIONES BÁSICAS
}

\author{
Ana Isabel Mora Vargas \\ Docente en la Escuela de Formación Docente
}

Recibido 30-VIII-2004 • Aceptado 7-IX-2004

\begin{abstract}
Resumen: En este trabajo se ofrecen orientaciones básicas para que el estudiante del área de educación incorpore la actitud hacia la investigación en su formación académica y la pueda transferir posteriormente a su desempeño profesional. Se proponen cuatro temas: la relación docencia-investigación, el diagnóstico educativo, esquema del informe de investigación y una guía para citar las referencias bibliográficas. Cada tema recoge la experiencia acumulada de la autora en la labor docente y de investigación en el nivel de educación superior, se ofrece un fundamento teórico básico y se sugieren actividades de aplicación.
\end{abstract}

Palabras clave: Docencia-investigación, diagnóstico educativo, informe de investigación, referencias bibliográficas.

Abstract: This article offers guidelines so that education students can incorporate a positive attitude towards research in their academic experience. In this way, they would be able to later transfer such attitude to their professional performance. Four topics are proposed in this article: the teachingresearch relationship, the educational diagnosis, the structure of a research report, and a guide for citing bibliographical references. Each of the topics summarizes the author's experience both as a teacher and researcher at the higher education level. Some basic theoretical foundations and suggested activities for practical application are also offered.

Keyword: Teaching-research, educative diagnosis, research report, bibliographical references.

\section{Introducción}

El propósito de este artículo es resaltar la relación de la docencia y la investigación, y ofrecer orientaciones básicas para iniciar un proyecto de investigación en el ámbito educativo. El artículo se fundamenta en una cuidadosa revisión bibliográfica acerca de tema y, muy especialmente en los datos recopilados por la autora durante más de quince años de experiencia como docente e investigadora en el campo educativo.

Está dirigido especialmente a estudiantes que pretenden hacer de la enseñanza su profesión. La investigación está presente a lo largo de su formación profesional, en diversos trabajos que se asignan en los cursos de la carrera, se presenta en distintos niveles de profundidad y complejidad. La gran mayoría de los cursos de una carrera incluyen actividades que refieren a investigaciones bibliográficas; luego se va profundizando en el análisis de situaciones educativas, observaciones de aula $\mathrm{y}$, en cursos avanzados se plantean prácticas supervisadas o investigaciones cortas. Estas últimas requieren del planteamiento de un proyecto con las implicaciones que eso significa: búsqueda y sistematización de información bibliográfica, identificación de un tema y su relación con la docencia, definición del problema y objetivos, elabo- 
ración de un marco conceptual y definición del procedimiento metodológico, entre otros aspectos. Las inquietudes del estudiantado en este tema son diversas y no es posible abarcarla en un solo artículo, razón por la cual se invita a consultar el artículo denominado "Guía para elaborar una propuesta de investigación”, en este mismo número de la revista educación.

Dentro de los principales retos para el cambio educativo se destaca la concepción educativa centrada en el ser humano y con énfasis en el desarrollo de procesos cognitivo y actitudinales. Desde hace una década se menciona este planteamiento teórico en las políticas educativas y curriculares de Ministerio de Educación Pública; no obstante, el cambio no es visible en la práctica, dado que las estrategias didácticas continúan estimulando la recepción de contenidos fragmentados. Las indagaciones empíricas, al respecto, señalan que el cambio se debe plantear y desarrollar desde las bases; es decir, desde quienes diseñan los planes de estudio de formación docente, quienes los ejecutan y quienes reciben esa formación, dado que se convierte en un efecto multiplicador que, posteriormente, se traslada a las aulas. El cambio no es solamente una adecuación de contenidos curriculares sino también de innovación en las técnicas de comunicación didáctica y, en la formación actitudinal hacia la enseñanza y el aprendizaje.

En los planes de estudio de las carreras de educación, se plantean, explícita o implícitamente, ejes transversales; es decir, que la formación en valores y actitudes están presentes en el diseño y ejecución de los planes de estudio. La investigación aunque no es señalada como un eje transversal, debe considerarse como tal y en este caso, como señala Yus (1998) se debe tratar metodológica y didácticamente desde la dimensión teórica, personal y social.

En un enfoque educativo con énfasis en procesos cognitivos y actitudinales, la investigación es uno de los elementos básicos para estimular la experimentación, la creatividad, el análisis y la reflexión; por lo tanto, el docente y la docente que desarrolla estas habilidades las puede transmitir al estudiantado. En este sentido, la investigación juega un papel preponderante en la forma de adquirir, interiorizar, relacionar, comparar y equilibrar los conocimientos que propician los aprendizajes.

El documento se organiza en cuatro apartados: la relación docencia-investigación, lineamientos para el diagnóstico de necesidades educativas, esquema del informe de investigación y lineamientos para citar y presentar las referencias de información tanto bibliográficas como electrónicas.

\section{La relación docencia-investigación}

La relación docencia-investigación constituye el primer tema dado que se pretende invitar a la reflexión acerca de la importancia de la docencia y la investigación como funciones básicas en la formación del profesional en educación.

Tradicionalmente la investigación educativa ha estado en manos de investigadores que no son educadores. Ese distanciamiento entre quién investiga y quién es protagonista de lo que se investiga ha ofrecido aportes en el nivel general de la educación; no obstante, se requiere de un análisis más cercano a la realidad educativa. La creencia de que el educador no puede realizar investigación lo ha llevado a tomar resultados de investigaciones que resultan ajenas a la realidad de su práctica educativa.

En la formación integral de un educador deben estar presentes tres actividades básicas: la formación académica y personal, el desempeño docente y la investigación. La primera proporciona al estudiante los conocimientos, habilidades, valores y actitudes básicas para apropiarse del estilo docente que lo caracteriza como profesional. La segunda, permite que el futuro docente 
adquiera los conocimientos y habilidades propias del desempeño profesional que evidencien su forma lógica de pensar y actuar. La investigación como tercera actividad, se puede considerar como un eje transversal en su formación académica y profesional; está ligada directamente con las otras dos actividades. La investigación le permite al estudiante de educación apropiarse de técnicas y métodos científicos que fundamentan su formación y actuación profesional.

Desde esta perspectiva, la docencia se convierte en el objeto de investigación y la persona encargada de la docencia en el sujeto de investigación. Es decir, se debe desarrollar la actitud y la habilidad para convertirse en un docente-investigador de su propio desempeño profesional.

Para el desarrollo de este tema se plantean los siguientes objetivos y metas.

\begin{tabular}{ll}
\hline Objetivos & Metas \\
\hline $\begin{array}{l}\text { Reflexionar acerca de } \\
\text { la importancia de la } \\
\text { relación docencia- } \\
\text { investigación. }\end{array}$ & $\begin{array}{l}\text { Elementos que } \\
\text { fundamentan la } \\
\text { relación docencia- } \\
\text { investigación. }\end{array}$ \\
$\begin{array}{l}\text { Determinar las } \\
\text { características del } \\
\text { docente-investigador. }\end{array}$ & $\begin{array}{l}\text { Perfil del docente- } \\
\text { investigador. }\end{array}$ \\
\hline
\end{tabular}

La docencia y la investigación se retroalimentan mutuamente dando como resultado un mejoramiento en la calidad del trabajo en el aula. En este sentido, el docente aprovecha la investigación como el medio propicio para analizar las problemáticas que intervienen en el proceso de enseñanza y aprendizaje dentro y fuera de contexto áulico. La investigación también le permite comunicar y discutir los resultados y descubrimientos con el estudiantado o sus familiares. En consecuencia, los resultados de la investigación le ofrecen pautas, al docente, para elaborar el planeamiento curricular acorde con el contexto socio-cultural donde se va a desarrollar la acción educativa.
Se considera pertinente iniciar esta reflexión teniendo una idea del concepto de investigación, este término es de uso cotidiano, se emplea constantemente en la conversación informal o formal; por ejemplo: "voy a investigar si los estudiantes mejoran el rendimiento con una nueva estrategia didáctica"; "es necesario indagar lo que ha pasado con Luis que no ha vuelvo a clases" $y$ "he observado que " $x$ " comportamiento se presenta con frecuencia, después de clase de ..., voy a interesarme por averiguar qué pasa”. De hecho se realizan algunas estrategias de indagación, se elaboran explicaciones y conclusiones y se intentan mejoras. Estos acercamientos a procesos investigativos, muchas veces, quedan en las vivencias del momento, no se sistematizan los hallazgos y soluciones emprendidas.

En el intento de concebir la investigación como un proceso científico, se analizan concepciones del término presentados por diferentes autores tales como: AnderEgg (1971), Sabino (1986), Canales (1986), Kerlinger (1991) y Hernández (1999). De ese análisis se puede concebir la investigación científica como la actividad que permite obtener un conocimiento objetivo, sistemático y organizado respecto a ciertos elementos de la realidad. La actividad investigativa propicia, en primer lugar, un acercamiento del sujeto hacia el objeto que desea investigar y, en segundo lugar, una confrontación de la teoría elaborada con la práctica, dando origen a una nueva teoría.

La docencia, por su parte es "...la actividad o conjunto de acciones sistematizadas o interrelacionadas del docente y de los estudiantes, que se desarrolla, con el fin de lograr los objetivos propuestos, durante la apropiación de los contenidos planificados" (Arteaga, 2000:2). En la labor educativa, docente y estudiante se relacionan como participantes activos donde ambas partes son fuentes de información recíproca. En esta actividad se puede influir en la personalidad del grupo estudiantil y se pueden obtener diversas y valiosas formas de enseñanza y aprendizaje. En ello interviene 
fuertemente el estilo de enseñanza, el estilo de aprendizaje, los métodos y el contexto áulico. Para la persona encargada de la docencia, el estudiantado es el sujeto principal del proceso investigativo.

En todo proceso investigativo se pueden considerar diferentes fases o momentos que le dan continuidad; Sabino (1986) señala cuatro, a saber: lógico, metodológico, técnico y nueva elaboración de teoría. Estos momentos guardan secuencia y coherencia entre sí.

En el momento lógico el investigador organiza y sistematiza sus inquietudes, sus preguntas y ordena los elementos que constituyen su punto de partida. Es, en este momento, donde se define el objeto de estudio; es decir, el investigador concreta qué es lo que quiere saber y respecto a qué hechos. Se determinan los problemas, objetivos y metas $\mathrm{y}$, se elabora un acercamiento al marco conceptual. Todo este proceso debe guardar una coherencia lógica entre lo que se formula y el fundamento teórico. En esta fase se parte de un acercamiento del sujeto al objeto, de la teoría a la práctica.

El momento metodológico es cuando el investigador plantea una estrategia para estudiar los hechos. En este sentido, formula una modelo operativo que le permita acercase al objeto de estudio y conocer sus manifestaciones, así como determinar los métodos y estrategias específicas, que han de servir para desarrollar la investigación y que ofrezcan la posibilidad de confrontar la teoría con la práctica. En esta fase se debe poner especial atención a la elaboración de sistemas de comprobación lo más objetivos posible.

Una vez que se eligen los métodos y las estrategias generales se pasa al momento técnico, donde se seleccionan las formas y procedimientos concretos que ayudan a recolectar y organizar la información. La distinción entre estos dos momentos es básicamente pasar de la elaboración del diseño metodológico a la aplicación de técnicas para obtener la información necesaria que permitirá dar respuesta a los objetivos planteados.
El momento final se caracteriza por la nueva elaboración teórica en función de los datos recopilados en la fase anterior. Es un proceso dialéctico, en la primera fase se parte del sujeto al objeto, de la teoría a la práctica y al finalizar el proceso se vuelve de la práctica a la teoría, del objeto al sujeto. En otras palabras, el docenteinvestigador en primer lugar tiene un contacto cercano con la experiencia que está investigando. Luego reflexiona y valora los hallazgos, explicándolos e interpretándolos en relación con la teoría y la coherencia lógica y técnica. Como resultado de ese proceso se elabora una nueva teoría; esto se da porque el docente-investigador logra hilar, desde su trabajo de investigación, una perspectiva teórico-práctica a partir de la cual comprende las manifestaciones del objeto de estudio.

En la reflexión acerca de la importancia de la relación docencia-investigación se señalan algunos aspectos a considerar en el proyecto educativo de las escuelas formadoras de docentes. Estos se han elaborado con base en ideas de autores como Villanueva (2002), Arteaga (2000), Morán, (2002) y Sabino (1986): y de la experiencia de la autora en la docencia y en la investigación en el ámbito de la planificación curricular.

- La investigación debe considerarse como premisa fundamental en los planes de estudio de formación docente para elevar la calidad académica. La investigación educativa se entiende como proceso de formación, como actividad de aprendizaje y como instrumento de indagación de la docencia (Sabino, 1986).

- Es necesario que en la formación de formadores se establezcan vínculos entre saber y pensar, entre quienes investigan y quienes enseñan en las carreras de educación.

- La investigación como método o procedimiento de aprendizaje le permite al docente conocer y analizar su 
propia práctica y, así, estar en condiciones de transformarla. También le permite asumir los contenidos de su disciplina de una manera científica; de igual manera desarrollar habilidades para propiciar en sus estudiantes el espíritu creativo y de indagación.

- La investigación educativa como sustento de una estrategia institucional de autoformación del docente en servicio tendiente a elevar la calidad de la educación.

- En la investigación educativa se entienden como procesos inseparables la formación, el aprendizaje y la indagación.

- En la investigación educativa se pueden distinguir tres orientaciones analíticas Sabino (1986):

- Procedimiento pedagógico, tiene como finalidad propiciar y fomentar el espíritu creativo en un proceso de enseñanza y aprendizaje.

- Praxis pedagógica, se orienta al análisis de fondo de determinadas problemáticas detectadas en la realidad educativa con el propósito de transformarlas y ofrecer soluciones viables.

- Investigación científica, consiste en conceptuar la investigación como un proceso formal que conduce a la producción de conocimientos nuevos. En esta orientación se supone un vasto conocimiento de las variables a investigar de cuerdo con la interpretación y explicación del objeto de estudio. Como actividad científica debe cumplir ciertas exigencias propias de este proceso, tales como: indagación metódica y sistemática, fundamentación teórica, sistemas de validación de la información y la producción de nuevos conocimientos.

- La investigación como herramienta intelectual básica, tanto para docentes como para estudiantes, permite enfrentar con mayores criterios teóricos y metodológicos su proceso formativo y, posteriormente, su propio ejercicio profesional.

Se ha mencionado anteriormente que el trabajo cotidiano del docente lo identifica como un indagador empírico de su propio quehacer profesional. $\mathrm{Al}$ respecto cabe preguntarse, qué tipo de formación académica y personal requiere para convertirse en docente-investigador.

En consecuencia con esos cuestionamientos, se propone a los estudiantes, realizar una indagación para determinar las características que se deben tomar en cuenta en la formación de docentes. La caracterización puede abarcar dos áreas: la académica y la personal. En la académica se obtiene información acerca de los conocimientos, habilidades y destrezas que deberían considerarse en un plan de estudio. En el área personal se puede incluir la información relacionada con los valores y actitudes que se deben fomentar en un docente-investigador. A continuación se ofrecen actividades que puede realizar para contribuir al conocimiento y desarrollo de este tema.

Actividades de aplicación

\begin{tabular}{ll}
\hline Metas & Actividades \\
\hline $\begin{array}{l}\text { Aporte a la reflexión } \\
\text { acerca de la relación } \\
\text { docencia-investigación }\end{array}$ & $\begin{array}{l}\text { Entrevistar a docentes } \\
\text { para obtener informa- } \\
\text { ción acerca de: }\end{array}$ \\
Perfil del & La importancia de la \\
docente-investigador & relación docencia- \\
& investigación, y cómo \\
& se refleja en el desa- \\
& rrollo del currículum \\
en el aula. & Las características \\
& de formación acadé- \\
& mica y personal de \\
& un docente- \\
& investigador.
\end{tabular}




\section{Diagnóstico de necesidades educativas}

Es difícil determinar el qué, por qué y para qué de una investigación; por lo tanto, partir de un diagnóstico de necesidades, sin duda, es una estrategia acertada para iniciar el trabajo de indagación. Este proceso, al principio, puede parecer disperso y sin unidad conceptual ni metodológica y poco a poco se va consolidando en torno a una propuesta de investigación acerca de un determinado tema. El diagnóstico se puede considerar como un proceso para encontrar las causas primarias y las interrelaciones que permiten el planteamiento de un tema de investigación que poco a poco se va perfilando en un proyecto.

Para el desarrollo de este tema se plantean los siguientes objetivos y metas

\begin{tabular}{ll}
\hline Objetivo & Meta \\
\hline - Determinar los & $\cdot$ El tema y problema \\
elementos que & de investigación: \\
componen el & antecedentes y \\
diagnóstico de & justificación. \\
necesidades en & \\
el ámbito educativo. & \\
\hline
\end{tabular}

En el ámbito educativo y curricular el diagnóstico se puede concebir de diferentes maneras (Delgado Morera, 1995; Trigo, 1996, Sabino, 1996 y estudiantes de educación de la Universidad de Costa Rica): Se sistematizan algunas de las premisas que fundamentan este concepto:

- Es el proceso de determinación de los hechos que deben ser tomados en cuenta al adoptar decisiones tanto en el diseño de plan de estudios como en el planeamiento y desarrollo del curriculum.

- Es la base del planeamiento curricular, éste requiere sistematización, orden y secuencia.

- Es un análisis de los elementos que componen la situación de aprendizaje.
- $\quad$ Es un proceso por medio del cual se determinan los hechos que deben ser valorados con el propósito de adoptar decisiones curriculares.

El diagnóstico en la elaboración del currículum trata de encontrar información para dar respuesta a problemas relacionados con: el rendimiento o logro del aprendizaje, los alumnos como sujetos de la enseñanza, el ambiente extraescolar, la puesta en práctica del currículum, entre otros. En consecuencia, el propósito del diagnóstico es analizar las necesidades de aprendizaje, sus antecedentes, conceptos y perspectivas que permiten proponer lineamientos para su adecuación.

El docente no debe diagnosticar por diagnosticar, sino para la detección y búsqueda de problemas que afectan el proceso de enseñanza y aprendizaje; $\mathrm{Al}$ respecto, se pueden distinguir diagnósticos formales e informales o menos rigurosos. El diagnóstico evaluativo con objetivos definidos constituye un diagnóstico formal, que se enmarca dentro del paradigma cualitativo o cuantitativo o una combinación de ambos. En este caso, quien investiga emplea técnicas cualitativas o cuantitativas para recolectar la información, tales como: conversaciones abiertas en el aula, preguntas y temas abiertos, relatos e incidentes ocurridos, registro de debates, registro de lectura y redacción, observación y registro del rendimiento, ejercitación y tareas especiales, test sociométricos, cuestionarios (estructurados, semiestructurados y abiertos), entrevistas abiertas, entrevistas "en profundidad", discusiones grupales, talleres de análisis y reflexión, análisis de contenidos y otras técnicas que considere pertinente de acuerdo con el objeto de estudio.

Se pueden realizar diferentes tipos de diagnóstico, en el papel del docenteinvestigador interesa resaltar el diagnóstico de necesidades educativas que proporciona información en relación con el tipo de ser humano, de sociedad y conocimientos que sirven de base para la planificación 
curricular. Es importante tomar en cuenta que el elemento clave del diagnóstico educativo es la práctica educativa relacionada con las necesidades reales y sentidas por la comunidad educativa. Por necesidad debe entenderse el estado de la persona que posee una carencia de algo y que requiere de soluciones viables. La necesidad puede clasificarse en primarias relacionadas con el mantenimiento de la especie humana y en secundarias o superiores propias del intelecto y la conciencia. También se clasifican en objetivas, subjetivas, materiales e ideales (Villarini, 1996, Trigo, 1996). En el ámbito educativo las necesidades se reflejan en la motivación que son el impulso básico para la adquisición del aprendizaje; de manera que, la satisfacción de las necesidades educativas contribuye a la formación integral de los educandos.

Esta actividad indagatoria se puede desarrollar en diversas áreas, según los estudiantes de la Escuela de Formación Docente de la Universidad de Costa Rica, entrevistados para este fin, al docenteinvestigador le interesan especialmente las áreas: geográfica, histórica, socioeconómica y educativa que le ofrecerán elementos claves para el planeamiento curricular y la puesta en práctica del currículum en el aula. Se señalan algunos elementos básicos de considerar en el diagnóstico de necesidades educativas (Navarro, 1994):

\section{Geográfico}

Localización del centro educativo (provincia, cantón, distrito, caserío). También los límites, el relieve, el clima, la hidrografía, entre otros.

\section{Histórico}

Origen de la comunidad, antecedentes de los pobladores, ciudadanos destacados, origen del nombre del Centro Educativo, etc.

\section{Socioeconómico}

Generalidades de la población, sexo, servicios comunales, nivel de escolaridad, costumbres, tradiciones, manifestaciones artísticas, problemas particulares y comunales.

\section{Educativos}

Matrícula efectiva de la institución, porcentaje de promoción, deserción, asistencia, rendimiento académico, niveles escolares, características según sexo.

Otros elementos: organizaciones, comités, mobiliario, material didáctico, equipo, etc.

La información que se recopila del diagnóstico de necesidades educativas se organiza y estructura con el propósito de explicar los hallazgos de la investigación. La finalidad de realizar un diagnóstico es conocer la problemática educativa e identificarse con alguna problemática que permita plantear un proyecto de investigación. Es necesario señalar que no todas las problemáticas detectadas en un diagnóstico reúnen la condición de viabilidad; por lo tanto, el diagnóstico también es una herramienta para definir y delimitar el tema de investigación.

Las variables de información que se consignan seguidamente se han elaborado con base en Navarro (1994) y la experiencia de la autora al conducir procesos de investigación con estudiantes tanto en los cursos iniciales de la carrera como con estudiantes que preparan proyectos de trabajos finales de graduación. Las variables deben responder a las necesidades particulares de la zona, de la comunidad educativa, de la situación que se desea diagnosticar y, por su supuesto, a los intereses e inquietudes de la persona que realiza el estudio.

\section{Algunas variables para un diagnóstico de necesidades educativas}

Cada situación educativa es diferente por lo que estas variables solo deben tomarse como una guía general, quien investiga 
debe conocer el contexto donde va a realizar el diagnóstico y definir sus propias variables. A continuación se citan cuatro categorías de variables: la comunidad, la institución educativa, el estudiantado y la docencia.

\section{En relación con la comunidad}

Características de la comunidad:

- Físicas y geográficas

- Condiciones socio económicas y culturales

- Políticas comunales

- Políticas económicas

- Valores, creencias, costumbres

- Relación escuela-comunidad

- Fuentes de trabajo

\section{En relación con la institución educativa}

Características de la institución educativa:

- Infraestructura

- Equipo y mobiliario

- Servicios escolares

- Relaciones humanas

- Filosofía y objetivos de la institución educativa

- Situación geográfica de la institución

\section{En relación con el estudiantado}

A manera de ejemplo, se citan las siguientes categorías de información:

\section{Situación de los alumnos}

Aspecto físico

Desarrollo corporal

Destrezas

Estado de salud

Características físicas en las tareas de aprendizaje

\section{Aspecto socio emocional}

Estado emocional

Relaciones interpersonales

Relaciones familiares

\section{Aspecto intelectual}

Pautas para el desarrollo intelectual

Posibilidades de pensamiento lógico y

abstracto

Desarrollo del lenguaje

\section{Personalidad}

Concepto de sí mismo

Valores, creencias e ideales

Normas de moral y comportamiento

Actitudes

Motivaciones, exigencias y necesidades

\section{Características socio económicas}

Nivel socioeconómico

Situación familiar

\section{Bases para un nuevo conocimiento}

Nivel de conocimientos básicos

Grado de comprensión y manejo de conceptos

Habilidades específicas

Técnicas y hábitos de estudio y comprensión

\section{En relación con la docencia}

Al igual que en las anteriores categorías se citan algunas variables que pueden ser susceptibles de diagnosticar.

\section{Aspecto socio emocional}

Estado emocional de la persona encargada de la docencia

Relaciones interpersonales

Relaciones familiares

\section{Personalidad}

Concepto de sí mismo

Valores, creencias e ideales

Normas de moral y comportamiento

Actitud hacia la docencia

Disposición hacia la atención de nece-

sidades educativas especiales

Sensibilidad hacia la diversidad multicultural 


\section{Estilo de enseñanza}

Dominio del contenido

Estrategias metodológicas y didácticas

Criterios para la evaluación

Manejo del tiempo

Manejo de límites

Estrategias para la adecuación curricular

El diagnóstico enfoca necesidades específicas que son determinadas por el investigador o investigadora. Se invita al estudiante a consultar otros lineamientos para la elaboración del diagnóstico; al respecto, Delgado en su libro "Elaboración de Proyectos en Centros Infantiles" (1998), ofrece una guía que incluye sectores tales como: comunidad, infraestructura, financiamiento, recursos humanos, currículum, administrativo y relaciones; en cada área se presentan diferentes variables a considerar e indicadores de las mismas. Por ejemplo, en el sector currículum, la autora señala cinco áreas: plan de estudios y programa, horario escolar, material didáctico, métodos y técnicas y evaluación. Delgado sugiere la siguiente tabla para organizar e interpretar la información recopilada.

El diseño del diagnóstico, las técnicas para recopilar la información y la estrategia para sistematizar e interpretar la información no responden a un modelo determinado, pero sí deben tener orden, secuencia y coherencia entre las fases de diagnóstico. Aunque se dice que no existe un modelo único para realizar un diagnóstico si se pueden distinguir tres fases generales: preparación, realización y resultados. A continuación se señalan aspectos para cada fase, éstas han sido elaboradas con base en manifestaciones e inquietudes de estudiantes que realizan sus trabajos de graduación en el ámbito educativo en la Universidad de Costa Rica y que han sido recopiladas y sistematizadas por la autora. A continuación se ofrecen tres fase para el diagnóstico de necesidades educativas

\section{Fases para el diagnóstico de necesidades educativas}

Se presenta en tres fases: preparación, realización y resultados.

\section{Preparación}

En esta fase se deben considerar aspectos que pretenden acercar el sujeto al objeto de estudio. En este primer momento del diagnóstico, puede parecer que al inicio las ideas se presentan dispersas y muy generales, las cuales van logrando claridad hasta plantear un plan de acción.

\section{- Determinar los elementos claves del análisis}

Se empieza por analizar la finalidad que se persigue con el diagnóstico, si es potencialmente viable, quiénes pueden ser los informantes, cuál podría ser el objetivo del estudio.

\section{- Clarificar la importancia del diagnóstico}

Determinar cuál es el propósito del estudio, qué es lo que se pretende lograr. En este paso se debe obtener permisos necesarios para acceder a las fuentes de

\begin{tabular}{|l|l|l|l|}
\hline $\begin{array}{c}\text { Principales } \\
\text { problemas del sector }\end{array}$ & $\begin{array}{c}\text { Causas que originan } \\
\text { el problema }\end{array}$ & $\begin{array}{c}\text { Solución que requiere } \\
\text { el problema }\end{array}$ & $\begin{array}{c}\text { Alternativas posibles } \\
\text { para su solución }\end{array}$ \\
\hline & & & \\
\hline
\end{tabular}

Tomado de Delgado (1998). 
información, contar con la aceptación de los informantes para la eventual aplicación de instrumentos.

\section{- Aproximación inicial a las nece- sidades educativas}

Se realiza una indagación previa acerca de los problemas educativos que conduzca a la definición de variables de interés que permitan delimitar el alcance del estudio. Para iniciar esta reflexión se sugiere tomar en cuenta las variables para un diagnóstico de necesidades citadas anteriormente.

\section{- Describir a los sujetos de infor- mación}

Se define quiénes pueden ofrecer la información requerida y qué tipo de información pueden ofrecer en relación con las variables de estudio dónde ubicarlos y cómo acceder a la información.

\section{- Plan de acción}

Este paso requiere de tres acciones fundamentalmente:

a. Elaborar, por escrito, la propuesta que se va a llevar a cabo, donde incluye el propósito, los objetivos, las variables del diagnóstico y una descripción de la estrategia a seguir.

b. Se selecciona el tipo de instrumentos idóneo para recopilar la información como por ejemplo, cuestionarios, entrevistas, hojas de cotejo, recopilación de textos, documentos, etc. Además se determina el tipo de ayuda o apoyo que se requiere para realizar el diagnóstico.

c. Se gestionan los permisos, autorizaciones (si son necesarios) para acceder a la información de documentos confidenciales, de aplicación de cuestionarios en horario de trabajo, entre otras situaciones. Hasta aquí se puede decir que se tiene un proyecto de diagnóstico, el paso siguiente es realizar el diagnóstico.

\section{Realización del diagnóstico}

Una vez preparado el plan de acción y acordada la puesta en práctica se inicia la de desarrollo del mismo. Aquí es necesario que la persona que investiga tenga claro qué tipo de diagnóstico va a realizar, si se trata de un diagnóstico informal el instrumento para recopilar la información es sencillo y solo requiere el visto bueno del docente del curso. Si por el contrario es un diagnóstico formal, los instrumentos para recopilar la información requieren de normas de validez y confiabilidad. Se sugiere, además de la asesoría del docente del curso, consultar referencias bibliográficas como por ejemplo, Hernández y Kerlinger para fundamentar este aspecto en investigaciones cuantitativas y Taylor y Bogdan para estudios de tipo cualitativo.

\section{Resultados}

Finalmente, se realiza un análisis de los datos de manera que permita dar respuesta a los objetivos planteados y ofrecer alternativas de solución a los problemas encontrados. Los resultados se pueden organizar en categorías de información cualitativa y en tablas y gráficos con porcentajes cuantitativos.

Es importante señalar que el diagnóstico de necesidades en este artículo tiene un carácter menos riguroso, se plantea con el fin de obtener claridad en la problemática educativa de una situación particular. Esto le permite al estudiantado identificar algún tema de su interés para plantear un problema de investigación; este es el primer paso para plantear un proyecto de investigación. 
Actividades de aplicación

\begin{tabular}{|c|c|}
\hline Metas & Actividades \\
\hline $\begin{array}{l}\text { El tema y problema de } \\
\text { investigación: } \\
\text { antecedentes y } \\
\text { justificación }\end{array}$ & $\begin{array}{l}\text { Elaborar el diseño del } \\
\text { diagnóstico } \\
\text { Describir las fases } \\
\text { del diagnóstico: } \\
\text { - Preparación } \\
\text { - Realización del } \\
\quad \text { diagnóstico } \\
\text { - Resultados }\end{array}$ \\
\hline & $\begin{array}{l}\text { Plantear el tema y } \\
\text { problema de investiga- } \\
\text { ción a partir de los re- } \\
\text { sultados del diagnóstico }\end{array}$ \\
\hline
\end{tabular}

\section{Esquema del informe de investigación}

Este apartado contiene elementos para estructurar y organizar una propuesta de investigación. Una propuesta es un planteamiento en construcción que está sujeto a ajustes antes de ejecutar el proyecto; por lo tanto, los elementos de la propuesta deben guardar secuencia y coherencia entre sí, de manera que permita valorar la pertinencia y viabilidad del proyecto.

El documento escrito de una propuesta de investigación debe ser fácil de leer, breve, conciso pero completo, con lenguaje simple. Organice el informe utilizando un esquema con título y subtítulos. El informe contiene al menos, la portada, tabla de contenido, introducción general al trabajo y tres capítulos: el problema, el marco conceptual y el procedimiento metodológico.

\section{Portada}

Contiene tres elementos básicos: el título, el nombre de la persona y la fecha. También se puede agregar el nombre de la institución, el nombre del curso y otros datos que la persona considere pertinente.

\section{Título}

Se refiere al nombre de la propuesta o anteproyecto, resume el tema de investigación de forma global. Se debe evitar el uso de títulos extensos y de términos relacionados con la metodología, lugar donde se va a desarrollar o la población meta como por ejemplo: "análisis del rendimiento académico de los estudiantes de sexto grado en la Escuela XX", del circuito $\mathrm{N}^{\circ}$ de la Dirección Regional de San José; un título como ese resulta muy largo y no expone claramente el objeto de estudio: El título debe ser corto e impactante, que invite a la lectura del documento, la elección del mismo depende del objeto de estudio y las variables de investigación. Por ejemplo si el objeto de estudio es el rendimiento académico podría resaltarse el objeto de estudio y las variables principales. Algunos ejemplos: "Rendimiento académica: su relación con el estilo de enseñanza y aprendizaje", "El rendimiento académico como causas de la deserción estudiantil en sétimo año".

\section{Tabla de contenido}

Indica los títulos y subtítulos principales del trabajo y el número de página.

\section{Introducción}

Se presenta una visión global del tema de manera que logre interesar al lector. Una forma de iniciar la introducción es explicar el tema de investigación, continuar exponiendo, en forma breve, la importancia y justificación del tema, el propósito de la investigación; es decir, indicar qué es lo que se pretende hacer y lograr con la investigación. También es necesario señalar la forma en que se va a organizar y estructurar el documento.

\section{Capítulo 1: El problema}

Se introduce el capítulo, explicando el tema y la delimitación del estudio. En un segundo apartado se puede ubicar los antecedentes y justificación y seguidamente el planteamiento de problemas, objetivos y metas. Se citan algunos aspectos básicos de este capítulo; no constituye un formato a seguir para el documento escrito. 


\section{Tema}

Identifica el objeto de estudio. El objeto se refiere al qué se va a investigar. También en el tema se especifica el área y el ámbito de investigación. Ejemplo: rendimiento académico en matemática en la educación secundaria. En este caso el objeto de estudio es "rendimiento académico", el área "matemática" y el ámbito "educación secundaria".

\section{Delimitación del tema de investigación}

Retoma el tema de investigación y lo relaciona con el propósito de investigación. Ejemplo: "plan para el mejoramiento del rendimiento académico en matemáticas en educación secundaria”.

\section{Problema(s) o preguntas de investigación}

A partir de la delimitación del tema se desglosan los aspectos relevantes en problemas o preguntas de investigación.

\section{Objetivo general}

Qué se propone hacer para lograr el propósito de la investigación.

\section{Objetivos específicos}

Cómo se propone desglosar el objetivo general para obtener la información y analizarla.

\section{Metas}

Constituyen acciones concretas, productos que se obtienen a partir de los objetivos específicos. Se plante por lo menos una meta para cada objetivo específico.

\section{Capítulo II: Marco conceptual}

En una propuesta de investigación se presenta un acercamiento al marco conceptual, dado que éste se construye paralelo al desarrollo de la proyecto de investigación. Por lo tanto, en este capítulo se presenta un esquema que permita explicar cómo se organiza y estructura el primer acercamiento al marco conceptual. Debe ofrecer una visión global de los conceptos, principios o tendencias teóricas que fundamentan las variables de estudio y su interrelación.

Con respecto a las referencias bibliográficas es necesarios corroborar: que las citas estén respaldadas en la bibliografía, que esté escrita correctamente y que las referencias de información bibliográfica sean suficientes y pertinentes para valorar la fundamentación teórica de la propuesta de investigación. En el último tema de este artículo se ofrecen lineamientos para citar y plantear las referencias bibliográficas.

\section{Capítulo III: Procedimiento metodológico}

En este apartado, quien investiga debe determinar y sustentar teóricamente el paradigma donde se enmarca la investigación, el tipo, diseño, método de investigación y las técnicas para recolectar la información. También debe citar los sujetos de información, la manera cómo pretende analizar la información recopilada y elaborar un cronograma de actividades.

\section{Paradigmas de investigación}

Un paradigma está constituido por los supuestos teóricos generales, las leyes y las técnicas para su aplicación que adoptan los miembros de una determinada comunidad científica. Cuando el estudiantado da los primeros pasos para plantear una propuesta de investigación, ya cuenta con bases teóricas acerca de los grandes paradigmas: cuantitativo y cualitativo. Es importante señalar que actualmente se habla de un tercer paradigma: críticoreflexivo y otro en construcción, llamado "paradigma de cambio", que trata de integrar los tres anteriores (Guba, 1985 y Reichard, 1986). 
Para enmarcar la investigación en uno o más paradigmas, la persona que investiga debe tener muy claro el objeto de estudio, el propósito del mismo y una clara posición epistemológica. En consecuencia, si el propósito consiste en realizar mediciones y predicciones exactas acerca de las causas del rendimiento académico, entonces su investigación se enmarca en el paradigma cuantitativo. Este paradigma es preferentemente deductivo, se recolectan datos para evaluar modelos, hipótesis o teorías preconcebidas. También puede darse el caso que el propósito consista en describir e interpretar la situación de quienes participan; por ejemplo, entender los fenómenos sociales que causan el bajo rendimiento académico en matemáticas, desde la propia perspectiva de los participantes; en este caso la investigación se enmarca dentro del paradigma cualitativo. En este paradigma se estudian las personas en el contexto de su presente y su pasado; desde esta perspectiva se considera válido la información que proviene del interior del sujeto de investigación (Taylor y Bogdan, 1986).

La perspectiva de investigación cuantitativa enfatiza sobre lo exterior, es decir lo válido o externo, posible de observación. En el paradigma cualitativo, los hechos sociales se diferencian de los hechos de las ciencias físicas por considerar las creencias y las opiniones de quienes participan. Hayet señala, por ejemplo, “... que no deben ser definidos según lo que podríamos describir sobre ellos por los métodos objetivos de la ciencia sino por lo que piensa la persona que actúa" (en cita de Bourdieu, 1987).

\section{Los métodos de investigación}

Los métodos determinan el camino que se sigue para dar respuesta a los objetivos planteados en la investigación. Se fundamentan en supuestos epistemológicos lo que permite distinguir una relación con algún paradigma.
En el enfoque cuantitativo, el método científico predominante en las ciencias sociales es el hipotético-deductivo, basado en la probabilidad de hipótesis a partir del referente conceptual y empírico. En el enfoque cualitativo prevalece el método inductivo en investigaciones de estudio de casos, etnografías, entre otras.

\section{Diseño de investigación}

El diseño es el plan que elabora la persona que investiga con el propósito de obtener respuesta al problema planteado o comprobar las hipótesis de investigación.

En cuanto al diseño de investigación, Hernández (2003), señala: las investigaciones experimentales y las no experimentales.

\section{Técnicas de recolección de la información}

Las técnicas seleccionadas deben ser coherentes con el paradigma y el método de investigación. Algunas técnicas son pertinentes para recopilar información cuantitativa como la observación sistemática, la entrevista cerrada y el cuestionario y otras para información cualitativa, tales como la observación participante, la entrevista abierta y en profundidad, la entrevista biográfica entre otras. Por lo tanto, el investigador debe estar en constante revisión de los objetivos propuestos para seleccionar las técnicas adecuadas. Una vez seleccionada la técnica se elaboran los instrumentos; éstos pueden ser hojas de cotejo, guía de entrevista, encuesta o cuestionarios con preguntas cerradas, abiertas o abiertas y cerradas, pruebas escritas, bitácoras por citar algunos.

\section{Fuentes de información}

Indicar quienes son los informantes (maestros, estudiantes, documentos, u otros) y cuáles son los criterios de selección. 


\section{Análisis de la información}

Se describe, en forma general, cómo se propone analizar, interpretar y presentar la información recopilada para dar respuesta a los objetivos de la investigación.

\section{Referencias bibliográficas}

En el caso de una propuesta de investigación se incluyen solo las referencias bibliográficas que se citan en la propuesta de investigación, dado que todavía no ha realizado una revisión exhaustiva de las referencias bibliográficas sobre el tema que va a investigar.

Los datos de la referencia deben estar escritos correctamente (apellido, nombre, año, libro o artículo..., lugar, editorial, páginas..., según estilo que elija). En el siguiente punto se ofrecen lineamientos para citar y presentar las referencias bibliográficas.

La bibliografía se presenta ordenada alfabéticamente con sangría (5 espacios hacia la derecha a partir del segundo renglón de cada referencia). La persona que investiga debe elegir un estilo y mantenerlo durante todo el documento.

\section{Lineamientos para citar y presentar referencias: formato escrito y electrónico}

En este punto se hace referencia a dos aspectos, relacionados con la forma de presentar las citas de autor dentro del texto y, la forma de organizar y presentar las referencias bibliográficas al final del documento.

\section{¿Cómo citar las referencias bibliográficas?}

Las citas bibliográficas constituyen el reconocimiento al autor de una idea que se incluye, se discute o se compara en el texto de la propuesta de investigación que viene desarrollando el estudiantado. Las citas en el texto pueden ser directas, indirectas o auxiliares. Para las citas directas e indirectas el sistema APA (2002) no sugiere utilizar citas al pie de página.

Las citas directas se presentan dentro del texto del trabajo, utilizando el apellido del autor, la fecha de publicación y la página citada entre paréntesis. Se puede citar de diferentes formas, a continuación se ofrecen ejemplos:

Barrantes, (2000:86) señala que ser buen lector "no solo es leer rápido y comprender lo que se lee, sino saber escoger lo que se estudia".

Es importante saber cómo sintetizar ideas y emplear un lenguaje adecuado para expresarlas (Barrantes, 2000, p. 86).

Las citas indirectas son las que ha citado el autor que se está consultando, por ejemplo:

Según lo señalado por Littelejophn (2001), citado por Hernández (2003), “Todas las teorías aportan conocimiento y en ocasiones ven los fenómenos que estudian desde ángulos diferentes".

Las referencias auxiliares corresponden a comunicaciones personales o referencias de trabajos no publicados; por lo tanto no pueden incluirse en el capítulo Bibliografía y se indican en el texto con un llamado a pié de página.

\section{¿Cómo presentar las referencias bibliográficas?}

Para ordenar y presentar las referencias bibliográficas en un trabajo de investigación se debe adoptar algún sistema, lo importante es que todas las referencias se citen con un mismo estilo. Es importante señalar que la bibliográfica debe escribirse en orden alfabético y dejando sangría (5 espacios) hacia la derecha a partir del segundo renglón de cada referencia. La sangría es necesaria porque permite 
visualizar el apellido de los autores en orden alfabético.

El propósito de este documento es ofrecer lineamientos para presentar las referencias bibliográficas. Se utilizan como ejemplos diferentes tipos de biográficas (actas, gaceta, revistas, entre otras) por considerarse de uso frecuente por el estudiantado universitario. Para ello se han analizado las diferencias y semejanzas de diferentes estilos que se utilizan actualmente, se han adaptado las ideas de dos sistemas: Sistema de Bibliotecas SIBUMCE, Santiago, Chile y Sistema Harvard APA (American Psychological Association); se invita al lector a consultar estas fuentes para ampliar la información. Es importante indicar que los documentos no siempre tienen todos los datos que se requieren para citar las referencias bibliográficas; no obstante, se debe tratar de seleccionar referencias bibliográficas que contengan, al menos, los datos del formato básico.

Esta guía contiene ejemplo de cómo presentar las referencias bibliográficas: en formato escrito, en formato electrónico y las referencias comunicativas.

\section{Referencias en formato impreso}

El formato básico en una referencia bibliográfica escrita es: autor, año, título, lugar, editorial, páginas.

\section{- ACTAS}

Sesión $N^{\circ}$ 13-2004 (6 de diciembre de 2004). Comité Científico. Instituto de Investigación en Educación. Universidad de Costa Rica. Pp.1-5.

DATOS

Sesión y número de acta

Fecha

Título

Lugar

Páginas

\section{- ARTÍCULO DE DIARIO}

CON INDICACIÓN DE AUTOR

Alfaro Obando, Anabelle. (25, julio, 2005).

Dengue se combate en las casas. La

Nación. El País. San José, Costa Rica. P. 12A.

DATOS

Autor(es)

Día, Mes y Año

Título del artículo

Título del diario

Título del suplemento

Lugar de publicación del diario

Página específica del artículo, indicando

la sección o cuerpo del diario

SIN INDICACIÓN DE AUTOR

Prevén alza de café después de agosto. (25, julio, 2005). La Nación. Economía. San José, Costa Rica. P. 31A.

DATOS

Título del artículo Día, Mes y Año Título del diario Lugar

Páginas específicas del artículo

\section{- DICCIONARIO, ENCICLOPEDIA}

\section{ENCICLOPEDIA COMO \\ OBRA COMPLETA}

Fishbein, Morris. (1966). Ed. Enciclopedia Familiar de la Medicina y la Salud. Vol. 1. New York, Ediciones H.S Stuttman Co. P. 404.

DATOS

Editor(ed.)/compilador(comp.) Año

Título

Edición

Lugar

Editorial

Páginas 
Actitud física por el ejercicio. En: Fishbein, Morris. (1966). ed. Enciclopedia Familiar de la Medicina y la Salud. Vol. 1. New York, Ediciones H.S Stuttman Co. P. 241.

DATOS

Autor del Artículo Título del Artículo

Año En: Editor(ed.) Volumen Lugar Editorial

Páginas del Artículo

Artículo sin autor, debe iniciar por el titulo del articulo

DICCIONARIO COMO

OBRA COMPLETA

Real Academia Española. (1984). Diccionario de la Lengua Española (20 a ed., Vols. 1-2, p. 1416). España: Editorial España-Calpe.

DATOS

Editor(ed.)

Año

Título

Edición

Lugar

Editorial

Páginas

PARTE DE UN DICCIONARIO

Familia. (1984). En: Diccionario de la Lengua Española (20 ${ }^{\mathrm{a}}$ ed., Vols. 1-2, p. 1416). España: Editorial EspañaCalpe, p. 630.

DATOS

Término

Año

En:

Editor(ed.)

Edición
Páginas específicas de localización del

término

\section{- DOCUMENTO ANÓNIMO}

Anónimo. (2004). Las adecuaciones curriculares significativas. San José de Costa Rica. Ministerio de Educación Pública. P. 10.

DATOS

Autor (anónimo o título del trabajo)

Año

Lugar

Editorial

Número de edición

Páginas

\section{- GACETA}

La Gaceta Universitaria 10-2005. (30 de junio de 2005). Universidad de Costa Rica. Centro de Información y servicios técnicos del Consejo Universitario. Año XXIX. Periodicidad permanente.

DATOS

Título de la gaceta y número.

Fecha

Lugar de publicación

Editorial

Año (números romanos)

Periodicidad

\section{PARTE DE UNA GACETA}

Sesión 4973, artículo 2, del martes 10 de mayo del 2005. (2005). Proyecto de Ley. Ley para la inspección y regulación de los centros docentes privados. En: La Gaceta Universitaria 10-2005. (30 de junio de 2005). Universidad de Costa Rica. Centro de Información y servicios técnicos del Consejo Universitario. Año XXIX. Periodicidad permanente. P. 10. 
Número de sesión y artículo

Título del artículo

En: Título de la gaceta y número.

Fecha

Lugar de publicación

Editorial

Año (números romanos)

Periodicidad de publicación

Página

\section{- LIBRO O MONOGRAFÍA}

Chaves Salas, Lupita. (2004). Los procesos iniciales de lecto-escritura desde la filosofía del lenguaje integral: teoría y práctica. Costa Rica: I.I.M.E.C.

DATOS

Autor

Año

Título

Lugar

Editorial

Páginas Número de edición

\section{Capítulo de libro escrito por un autor diferente al de la obra}

Pérez Serrano, Gloria. (2000). Familia y Educación. Demandas Mutuas. En: Gurdián, Alicia. Una mirada crítica a la educación (209-245). San José, Costa Rica: Editorial de la Universidad de Costa Rica. Pp.

DATOS

Auto

Año

Título del capítulo

En:

Autor

Título

Número de edición

Lugar

Editorial

Páginas específicas del capítulo

\section{- LEY}

Ley $\mathrm{N}^{\circ} 2298$ de 22 de noviembre de 1958. (1965). Ley Fundamental de Educación. En Chacón Jinesta, Oscar. Código de Educación. San José, Costa Rica. Imprenta Trejos Hermanos. Pp. 333-345.

\section{DATOS}

Número de la ley Día, Mes y Año de aprobación Año de publicación Denominación Oficial Título de la publicación oficial

Lugar de publicación

\section{- MAPAS}

Mapa Físico Político de Costa Rica. (1998). Talleres Gráficos de Paulo Paoppi. Escala 1:456.757. Proyección con forma plana. Buenos Aires, Argentina, Ed. Talleres Gráficos de Paulo Paoppi. 1 mapa, col., $1.00 \times 1.20 \mathrm{~cm}$.

DATOS

Título del mapa

Año de publicación Autor(es) personal o corporativo

Número de edición Datos matemáticos: escala, proyección

Lugar de publicación

Editor

Número de mapas, color, dimensión

- MEMORIA DE CONGRESO (JORNADAS, SEMINARIOS)

Sexto Encuentro Nacional de Investigadores en Educación. (1992). San José, Costa Rica. 21 y 22 de mayo de 1992. Compendio de resúmenes. San José: Ministerio de Educación Pública, CENADI, p. 98.

DATOS

Nombre y título de la reunión Ciudad y país de la reunión Día, Mes y Año de la reunión 


\author{
Título distintivo del \\ documento que reúne todos los \\ trabajos \\ Lugar, Editorial y Año \\ Volumen \\ Total de páginas
}

\section{Parte de una memoria}

Quirós Tito, Mora, Ana Isabel. (1995). La relación entre el contenido y el método en la didáctica universitaria. En: Primeras Jornadas Internacionales de Pedagogía Universitaria. San José, Costa Rica, 7-11 septiembre, 1992. Memoria Primeras Jornadas Internacionales de Pedagogía Universitaria, Universidad de Costa Rica, Facultad de Educación, Departamento de Docencia Universitaria. Pp. 161-165.

\section{DATOS}

Autor(es)

Año de la publicación

Título de la contribución (ponencia)

En:

Nombre y título de la reunión

Ciudad y país (de la reunión)

Día, Mes y Año (de la reunión)

Título del documento que reúne los trabajos

Lugar, Editorial

Volumen

Páginas específicas de la contribución

\section{- REVISTA}

Educación. Revista de la Universidad de Costa Rica. San José, Costa Rica: Editorial de la Universidad de Costa Rica. 1977. Semestral

DATOS

Título de la revista Lugar de publicación

Editorial

Año de inicio Periodicidad

\section{Artículo de revista}

D’Agostino Santoro, Giuseppa. (1-2004). El alumno sobresaliente, su educación y la atención a sus necesidades especiales en el sistema educativo costarricense. En Educación. Revista de la Universidad de Costa Rica. San José, Costa Rica. 28(1): 145-155

DATOS

Autor

Fecha de publicación, mes y/o año

Título del artículo

En Título completo de la revista

Lugar de publicación

Volumen

Número (utilizar número arábigo) Dos puntos(:), páginas específicas del

artículo

\section{- TESIS, MEMORIAS} O SEMINARIOS

Mora Vargas, Ana Isabel. (1996). Relación entre el contenido programático de dos planes de estudio de la Universidad de Costa Rica (Contaduría Pública y Sociología) y las necesidades de formación para el desempeño profesional. Tesis para optar al grado de Magister Scientiae en Educación, Mención Investigación Educativa. Directora: Haydeé Mendiola. San José, Costa Rica, Universidad de Costa Rica. P. 300.

DATOS

Autor(es)

Año

Título

Seminario, Tesis, Práctica Dirigida, Proyecto, Memoria para optar al título de Nombre de quien dirige: Lugar Editorial Páginas 


\section{Referencias en formato electrónico}

El patrón básico para una referencia electrónica es: autor, (año), título, lugar, fecha de acceso a internet (mes, día, año), dirección disponible en Internet.

\section{- GACETA DIGITAL}

La Gaceta Universitaria (Derechos reservados 2005). Consejo Universitario. Universidad de Costa Rica. Recuperado el 20 de julio de 2005. http://cu.ucr.ac.cr

DATOS

Autor(es)

Año de derechos reservados:

Lugar

Fecha de acceso (recuperado) a Internet

Dirección disponible en Internet

PARTE DE UNA GACETA DIGITAL

Sesión 4973, artículo 2, del martes 10 de mayo del 2005. (2005). Proyecto de Ley. Ley para la inspección y regulación de los centros docentes privados. En: La Gaceta Universitaria (derechos reservados 2005). Consejo Universitario. Universidad de Costa Rica. Recuperado el 20 de julio de 2005. http://cu.ucr.ac.cr/gacetas/ gacetasb.php

Sesión, artículo, fecha

Año

Título

En:

Autor(es)

Año de derechos reservados:

Lugar

Fecha de acceso (recuperado) a Internet Dirección disponible en Internet

\section{- MENSAJE ELECTRÓNICO}

Vargas, Rebeca. Boletín Electrónico $\mathrm{N}^{\circ}$ 24-2005. Derechos reservados 2000. Instituto de Investigación en Educación, Universidad de Costa Rica. 22 de julio de 2005, 13:12. Dirección electrónica rvargasb@cariari.ucr.ac.cr.

DATOS

Autor(es)

Asunto

Año de derechos reservados:

Lugar

Fecha de acceso (recuperado) a Internet

Hora

Dirección electrónica del remitente

\section{- MEMORIA CONGRESO}

Congreso Internacional de Investigación Educativa. (2005). San José, Costa Rica. 1-4 febrero de 2005. Memoria. Disponible en el portal http://inie.ucr. ac.cr/congreso/memoria/index.htm.

DATOS

Autor(es)

Año

Lugar

Fecha del congreso

Título de memoria

Dirección disponible en Internet

\section{- REGLAMENTO}

Reglamento de Régimen Académico Estudiantil (aprobado en sesión 4632-03, 09-05-01. Publicado en el Alcance a la Gaceta Universitaria 03.2001, 25-05-01). En: Normativas Universitarias, orden alfabético. (derechos reservados 2005). Consejo Universitario. Universidad de Costa Rica. Recuperado el 19 de julio de 2005, de http://cu.ucr.ac.cr/normativ/ regimen academico estudiantil.pdf 
DATOS

Autor(es) o título del reglamento Aprobado sesión $\mathrm{N}^{\circ}$, fecha

Publicado en: $\mathrm{N}^{\mathrm{o}}$, fecha

En:

Carpeta electrónica dentro de un portal electrónico

Año de derechos reservados

Portal Electrónico

Fecha de acceso (recuperado) a Internet

Dirección disponible en Internet

\section{- $\quad$ REVISTA ELECTRÓNICA}

Actualidades Investigativas en Educación.

(Derechos reservados 2001). Instituto

de Investigación en Educación, Universidad de Costa Rica. Semestral. Recuperado el 22 de julio de 2005, de http://.inie.ucr.ac.cr, ISSN 14094703.

DATOS

Autor(es) o Nombre de la Revista

Lugar

Periodicidad

Fecha de acceso (recuperado) a

Internet

Dirección disponible en Internet

Registro internacional de la Revista

\section{Artículo de revista electrónica}

Mora Vargas, Ana Isabel. (2004). La Evaluación Educativa: Concepto, Períodos y Modelos. Revista Electrónica: "Actualidades Investigativas en Educación”, 4(1) Año 2004. Recuperado el 14 de marzo del 2005, de: http://revista.inie.ucr.ac.cr, ISSN 1409-4703.

DATOS

Autor(es)

Año

Título

Nombre de la revista.

Volumen, número

Fecha de acceso (recuperado) a
Internet

Dirección disponible en Internet

Registro internacional de la Revista

\section{- SITIO WEB}

Instituto de Investigación en Educación (INIE). Universidad de Costa Rica. 2000. San José, Costa Rica. Web: http://www.inie.ucr.ac.cr

DATOS

Autor(es)

Año

Título

Seminario, Tesis, Práctica Dirigida,

Proyecto, Memoria para optar al título de.....

Nombre de quien dirige:

Lugar

Editorial

Páginas

\section{Referencias comunicativas}

Las entrevistas, no se puede reproducir para el lector, entonces:

- No es obligatorio que se cite en la bibliografía final.

- $\quad$ Es conveniente citarlo en el texto a manera de comunicación personal. Ejemplo: (M. Rojas, entrevista personal, 25 de julio de 2004).

\section{Consideraciones finales}

Como consideraciones finales del artículo, se rescata la importancia de motivar y fomentar la actitud investigativa en docentes; es necesario investigar lo que el docente vive y conoce en relación con la cotidianeidad educativa.

En consecuencia, la persona encargada de la docencia se convierte en docenteinvestigador, quien busca posibles respuestas 
a cuestiones fundamentales de práctica educativa con el propósito de mejorar su desempeño laboral, se interesa en cómo lograr procesos significativos de aprendizaje, cómo enriquecer el desarrollo de los alumnos y las alumnas y del suyo propio, cómo mejorar el contexto sociocultural de aula, cómo atender la diversidad multicultural, cómo abordar las necesidades educativas especiales, entre otros aspectos. En este sentido, el diagnóstico de necesidades educativas, es parte esencial en la elaboración y la revisión del currículum, debe ser un elemento constante en la puesta en práctica docente.

Estructurar una propuesta de investigación y citar correctamente las referencias bibliográficas a veces resulta difícil para el estudiantado universitario, que se encuentra en cursos iniciales de su carrera; dado que es un trabajo cuidadoso y detallado. Es necesario mantener una secuencia y coherencia entre los elementos de una propuesta y de igual manera en una cita bibliográfica.

\section{Referencias bibliográficas}

Ander Egg, Ezequiel. (1971). Introducción a las Técnicas de Investigación Social, Ed. Humanitas, Buenos Aires.

APA. American Psychological Association. Manual de Estilo de Publicaciones. (2002). México D.F. Editorial Manual Moderno. 2a . Edición p. 433. http:// cu.ucr.ac.cr/gacetas/gacetasb.php

APA. American Psychological Association. Manual de Estilo de Publicaciones. (2002). México D.F. Editorial Manual Moderno. 2a Edición, p. 433.

Arteaga Herrera, José y Chávez Lazo, Eduardo. (2000). "Integración docente-asistencial-investigativa". Rev. Cubana Educ Med Super 14 (2), pp. 184-195.
Ato, M. (1992). Investigación en ciencias del comportamiento. Vol. I: Fundamentos. Barcelona: PPU.

Balluerka, N. (1999). Planificación de la investigación. Salamanca: Amarú.

Barrantes Echeverría, Rodrigo. (2000). Investigación. Un camino al Conocimiento. Un enfoque Cuantitativo y cualitativo. San José, Costa Rica: EUNED.

Bizquera, R. (1989). Métodos de Investigación Educativa. España: Ediciones CEAC.

Bunge, M. (1983). La investigación científica. Barcelona: Ariel.

Canales, Francisca; Alvarado, Eva y Pineda Elia. (1986). Metodología de la Investigación. México: Editorial Limusa.

Castro, J.A. y Salgado, A. (1989). Técnicas de investigación en las ciencias del comportamiento. (Vols. I y II). Salamanca: Dpto. de Publicaciones de la Universidad Pontificia de Salamanca.

Cazau, Pablo. (2001). "Investigación teórica e investigación empírica”. http:// www.galeon.com/pcazau/artmet invteo.htm

Cómo citar referencias bibliográficas. Sistema de Bibliotecas SIBUMCE, Santiago, Chile. bcontre@umce.cl. : http://members.tripod.com/sibumce/ citas.html

Delgado Morera, Elisa. (1998). Elaboración de Proyectos en Centros Infantiles. San José, Costa Rica: EUNED.

Dobles Izaguirre, Ma. Cecilia; Zúñiga Céspedes, Magaly y García Fallas, 
Jackeline. (1996). Investigación en Educación. San José, CR: EUNED, BID-FOD.

Elliott, John. (1997). La investigaciónacción en educación. Madrid: Ediciones Morata.

Gómez, Miguel. (1986). Elementos de Estadistica Descriptiva. San José, CR: EUNED.

Hernández, Roberto; Fernández, Carlos y Baptista, Pilar. (2003). Metodología de la investigación. México: Mc Graw-Hill.

Kerlinger, F.N. (1981). Investigación del comportamiento: Técnicas y metodología. México: Interamericana (edición original en inglés, 1979).

Kerlinger, Fred. (1991). Investigación del Comportamiento. México: Mc GrawHill Interamericana.

León, O.G. y Montero, I. (1997). Diseño de investigaciones. Madrid: Mc GrawHill (2a edición).

López, Ligia y Van Patten, Elia. (1980). Investigación bibliográfica y confección de trabajos escritos. San José, Costa Rica: EUNED.

Morán Oviedo, Porfirio. (2002). "La vinculación docencia investigación como estrategia pedagógica"

http://www.cesu.unam.mx/iresie/ revistas/perfiles/perfiles-ant/ $\underline{61-07 . h t m}$

Navarro, Carlos. (1994). "Prontuario para la investigación” (documento inédito).

Pérez Gómez, A. (1985). "Paradigmas contemporáneos de investigación didáctica”. En J. Gimeno Sacristán y A. Pérez Gómez. La Enseñanza: su teoría y práctica. Madrid: Akal Editor, pp. 95-138.

Pérez Serrano, María Gloria. (1990). Investigación-Acción Aplicaciones al campo Social y Educativo. San José, CR.: Dykinson.

Reichardt, Ch. y Cook, T. (1986). Hacia una superación del enfrentamiento entre los métodos cualitativos $y$ los cuantitativos en investigación. Madrid: Ediciones Morata, pp. 25-58.

Sabino, Carlos A. (1986). El proceso de investigación. Caracas: Panapo.

Taylor, S.J. y Bogdan, R. (1986). Introducción a los métodos cualitativos de investigación. La búsqueda de significados. Buenos Aires: Editorial Piados.

Trigo López, Julio. (1996). “Diagnóstico de salud y diagnóstico educativo. Un enfoque integral". Revista Cubana de Salud Pública 2.

Villanueva, P.; Monsell, A. y Cerda, C. (2001). "Líneas emergentes de investigación a partir de las reformas educativas en España en el ámbito de la "Pedagogía de la Diversidad". Departamento M.I.D.E.- Universidad de Valencia.

Villarini, Ángel. (1996). El currículo orientado hacia el desarrollo integral. Río Piedra, Puerto Rico: Biblioteca del Pensamiento Crítico.

Wittrock, Merlin C. (1989). La investigación de la enseñanza. Enfoques teorías y métodos (Tomo Y). México: ediciones PAIDOS.

Yus, Rafael. (1998). Temas transversales: hacia una nueva escuela. Barcelona, 
España: Editorial Graó, Serie Zubizarreta, Armando. (1969). La Aventura Transversalidad. del Trabajo Intelectual, Fondo Educativo Interamericano, Colombia. 
